\section{Effect on rats' straight alley performance of shifts in body weight as a function of method of weight maintenance*}

\author{
ELIZABETH D. CAPALDI $\dagger$ \\ Purdue University, Lafayette, Ind. 47907
}

Rats were maintained at either $80 \%$ or $95 \%$ of their initial ad lib weights or their expected weights. Weights were then shifted from $80 \%$ to $95 \%$. The effects of level of percentage body weight loss and the effects of shifting percentage body weight loss on straight alley performance were independent of method of weight maintenance.

An increasingly favored method of manipulating motivation in the rat is to vary percentage body weight loss. One question concerning the use of this measure is whether the loss should be measured from the animal's predeprivation weight (fixed percentage, FP) or whether adjustment should be made for the growth which would be expected to occur during the deprivation period (adjusted percentage, AP). Direct comparisons of the effects of FP and AP methods on performance have been made in the activity wheel (Moskowitz, 1959) and in the barpressing situation (Davenport \& Goulet, 1964; Marwine \& Collier, 1971). The main purpose of these studies was to determine which method of weight maintenance produces a more nearly constant motivational level. When an FP method has been employed, an increasing rate of performance over days, suggesting increasing motivation, has been obtained in the barpressing situation (Davenport \& Goulet, 1964 Marwine \& Collier, 1971), in the activity wheel (Moskowitz, 1959), and in the straight alley in some cases (Ehrenfreund, 1960, Figs. 2 and 3), but not in other cases (Ehrenfreund, 1960, Fig. 1; Ehrenfreund, 1971). When an AP method has been employed, a decreasing rate of performance over days, suggesting decreasing motivation, has been obtained in the barpressing situation (Davenport \& Goulet, 1964; Marwine \& Collier, 1971), but not in the activity wheel (Moskowitz, 1959) or the straight alley (Traupmann, 1971). Thus, the question of which method produces a more nearly constant motivational level appears open, but this question was not of major concern here.

One purpose of the present study

* The author is grateful to Joe Payne for experimental assistance in this investigation.

+ Requests for reprints should be sent to Elizabeth D. Capaldi, Dept, of Psy chology, Purdue University, Lafayette, Ind. 47907. was to determine if the effects of level of percentage body weight loss differ as a function of method of weight maintenance. Previous studies involving instrumental learning (Davenport \& Goulet, 1964; Marwine \& Collier, 1971) have compared FP and AP methods at only one level of percentage body weight loss. The present study compared $\mathrm{FP}$ and $\mathrm{AP}$ methods at two levels of percentage body weight loss. The second purpose of the present study was to evaluate the effects of shifting percentage body techniques. It has been reported that animals shifted from a high to a low deprivation run faster in the straight alley than animals trained only under Low deprivation (e.g., Zaretsky, 1966). It is possible that this effect may be influenced by method of weight maintenance. Specifically, if an FP method is employed, it is possible that in the second phase of such a study, when all groups are run under low percentage body weight loss, this low percentage body weight loss is actually a fairly high motivational level, if motivation increases over days with the FP method. Thus, animals trained previously under high percentage body weight loss could be expected to run faster than animals previously trained under low, because the testing situation is more similar to the training situation for the previously high percentage body weight loss animals. It could be expected, then, that a persisting effect of previous training under high percentage body weight loss would occur when the FP method is employed, but not when the AP method is employed. No previous study has employed the AP method and compared a group shifted from high to low motivation with a group maintained under low motivation.

\section{SUBJECTS}

The Ss were 48 naive male albino rats, 90 days old when purchased from the Holtzman Co., Madison, Wis. The Ss were fed ad lib for 30 days, at which time pretraining began. weight loss under the two different
APPARATUS

The apparatus was a runway painted a flat gray throughout. $208.1 \mathrm{x}$ $10.2 \mathrm{~cm}$, with $22.9-\mathrm{cm}$-high sides, covered with $1.3 \cdot \mathrm{cm}$ hardware cloth. A $20.3 \mathrm{~cm}$-long floor treadle suspended over a microswitch constituted the initial portion of the alley, and when the treadle was depressed by the weight of the rat, a .01-sec clock was started. The clock was stopped (start time) and another started when the rat interrupted a photobeam $5.08 \mathrm{~cm}$ beyond the treadle. The latter clock was stopped (run time) and a third one started when the rat broke the second photobeam located $132.1 \mathrm{~cm}$ from the first. Interruption of the third photobeam, $38.7 \mathrm{~cm}$ beyond the second, stopped the final clock (goal time). The third photobeam was located $7.5 \mathrm{~cm}$ in front of a teaspoon that was attached to the side of the goalbox and served as a foodcup. The teaspoon was $4.4 \mathrm{~cm}$ from the rear of the goal compartment. A manually lowered guillotine door, $27.8 \mathrm{~cm}$ from the rear of the goal compartment. served to confine the rat to the goa: area. The times from the three clochs were summed to obtain total time.

\section{PROCEDURE}

The rats were divided randomly into four experimental groups of $10 \mathrm{Ss}$ each and one control group of 8 rats. which was employed to obtain an estimate of growth during the course of the experiment. The control rats were fed ad lib throughout the experiment. The control rats ganed $50.4 \mathrm{~g}$ on the average. during the course of the experiment. On Days 1-3 of pretraining, the rats continued to be fed ad lib. The average weight of each rat over these 3 days was taken as its original ad lib body weight. The original ad lib weights of the five groups did not differ significantly ( $F=$ 1.56). The design of the experiment in Phase 1 was a 2 by 2 factorial combining two methods of weight maintenance (FP or AP) with two levels of percentage body weight $(80 \%$ or $95 \%)$. Beginning on Day 3 of pretraining, each animal in Group $80 \%$ FP was brought to and then maintained at $80 \%$ of its original ad lib weight $(79 \%-81 \%$ was an acceptable range), while each animal in Group 95\% FP was brought to and then maintained at $95 \%$ of its original ad lib weight $(94 \%-96 \%$ was an acceptable range). For animals in Groups $80 \%$ AP and $95 \%$ AP, an original ad lib weight was also computed. Beginning on Day 4 of pretraining, an acceptable weight range was computed for each rat in these groups on each day. For rats in Group $80 \%$ AP, the acceptable wejght range was $79 \%-81 \%$ of the rat's original ad lib weight plus $80 \%$ of the 


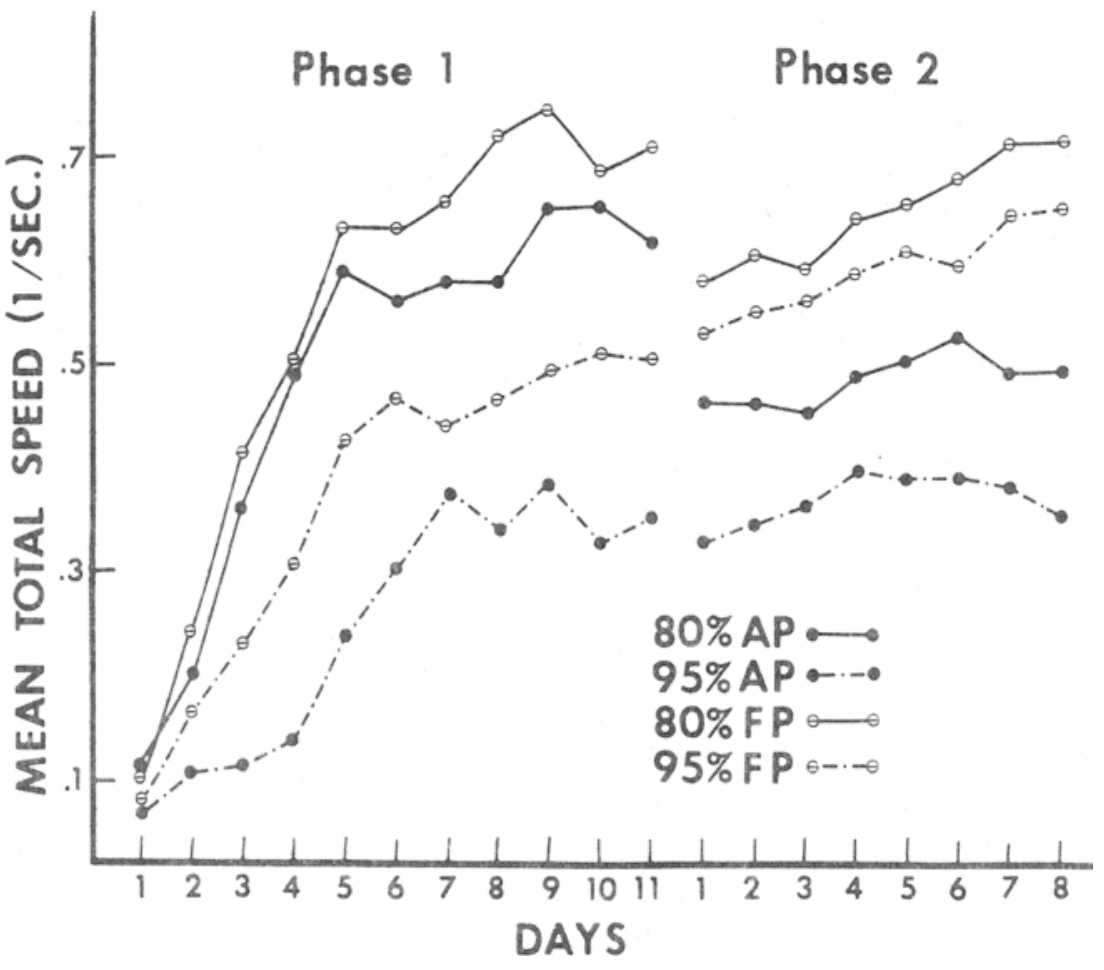

Fig. 1. Mean total speed for each group on each day of Phase 1 and Phase 2.

mean weight gain of the control rats on that day. For rats in Group $95 \%$ $\mathrm{AP}$, the acceptable weight range was $94 \%-96 \%$ of the rat's original ad lib weight plus $95 \%$ of the mean weight gain of the control rats on that day. By Day 16, all rats were within their appropriate weight ranges. On Days 16,17 , and 18 , each rat was handled individually for $90 \mathrm{sec}$ and was fed eight $.045 \mathrm{~g}$ Noyes pellets in its home cage $15 \mathrm{~min}$ after handling. On Day 19, experimental training began. On the first 2 days of experimental training, each rat received one rewarded trial, on the 3rd day, each rat received two rewarded trials, and on the next 10 days, each rat received three rewarded trials. There were thus 34 trials in Phase 1 , each trial being rewarded with eight .045-g Noyes pellets. On every trial, the rat was removed from the goalbox as soon as it had consumed all the pellets. After completion of Phase 1, animals in Group $80 \%$ AP were shifted to $95 \%$ AP. To do this, for each rat in Group $80 \%$ AP, $94 \%-96 \%$ of its original ad lib weight was computed and $95 \%$ of what the control rats had gained since Day 4 of pretraining was added to this weight. Thereafter, the acceptable weight range was determined each day in the same manner as it was determined for Group 95\% AP. The animals in Group $80 \%$ FP were shifted to $95 \%$ of their original ad lib weight $(95 \%$ FP). It took 8 days for the animals in these groups to attain their new weights. They were maintained at these weights for 2 more days, and then Phase 2 began. Phase 2 lasted 8 days (three trials per day), each trial being rewarded with eight pellets. In Phases 1 and 2 , animals were run in squads of four composed of one animal from each group, producing an intertrial interval of 6-8 min. Each squad was fed its daily ration at least $15 \mathrm{~min}$ after completion of its trials. During Phase 1 , three rats died, two in Group 95\% AP and one in Group 95\% FP, and their data were discarded.

\section{RESULTS}

All times were converted to speeds ( $1 / \mathrm{sec}$ ), and a 2 by 2 factorial between and within analysis for unequal ns was done on speeds in Phase 1 and Phase 2. As results were essentially the same in all alley sections, only total speeds are presented here. Figure 1 shows the mean total speed for each group on each day of Phase 1 and Phase 2 (the first four trials of Phase 1 were treated as Day 1, although these trials were actually spread over 3 days). As may be seen in Fig. 1, in Phase 1, $80 \%$ animais ran faster than $95 \%$ animals and animals maintained by the FP method ran faster than animals maintained by the AP method. Both of these effects were significant (level, $\mathrm{F}=45.61, \mathrm{df}=1 / 33, \mathrm{p}<.001 ;$ method, $F=8.45, \mathrm{df}=1 / 33, \mathrm{p}<.01$ ). The Method by Level interaction was not significant $(F=1.13, \mathrm{df}=1 / 33$, $p>.10)$. In addition, in Phase 1 , the effects of body weight level increased over days $(F=5.71, \mathrm{df}=10 / 330$, $\mathrm{p}<.001$ ). No other effects were significant in Phase 1. In Phase 2, all animals were run under $95 \%$ body weight. Animals previously trained under $80 \%$ body weight continued to run faster in Phase 2 than animals previously trained under $95 \%$ body weight $(F=4.31$, $\mathrm{df}=1 / 33, \mathrm{p}<.05)$. As may be seen in Fig. 1 , this effect appears to be larger for AP animals than for FP animals. However, the effect did not differ significantly as a function of method of weight maintenance $(F<1)$. Thus, it appears that the effects of shifting percentage body weight loss are independent of method of weight maintenance. As in Phase 1, in Phase 2, animals maintained by the FP method ran faster than animals maintained by the AP method $(F=20.16$, df $=1 / 33$, $\mathrm{p}<.001)$. In addition, in Phase 2 , the effect of method of weight maintenance increased over days $(\mathrm{F}=$ $2.93, \mathrm{df}=7 / 231, \mathrm{p}<.01$ ). As may be seen in Fig. 1, this effect was due to the FP groups increasing in speed over days (Group $80 \% \mathrm{FP}, \mathrm{F}=6.72, \mathrm{df}=$ $7 / 63, \mathrm{p}<.001$; Group 95\% FP, $\mathrm{F}=$ $5.69, \mathrm{df}=7 / 56, \mathrm{p}<.001$ ), while the AP groups maintained a constant speed $(F s<1)$. No other effects were significant in Phase 2 .

Marwine \& Collier (1971) reported a significant negative correlation between barpress rate and water ration (water was the reward and water deprivation was employed) for their FP group. Specifically, a decrease in water ration, which was necessary to keep rats at constant weight, was associated with an increase in barpress rate. Marwine and Collier suggested that the increase in barpress rate for their FP group might be partially attributable to this decreasing ration. In the present experiment, however, it was necessary throughout Phases 1 and 2 to consistently decrease the food ration for both Groups 95\% AP and 95\% FP in order to maintain them at the appropriate weight. In addition, the correlation between amount fed and speed was not significant on any day, or overall Phase 1 , or overall Phase 2 for either of these groups (or for the other two groups). For Group $95 \% \mathrm{FP}$, these correlations ranged from -.35 to .51 , with most being near zero. Thus, the increase in speed obtained here in Phase 2 for Group 95\% FP does not appear to be attributable to their decreasing food ration.

\section{DISCUSSION}

In the present study, the effects of level of percentage body weight loss and the effects of shifting percentage 
body weight loss were independent of method of weight maintenance schedule. Whether or not adjustment was made for growth, animals previously trained under $80 \%$ body weight ran faster in a test phase under $95 \%$ body weight than animals previously trained under $95 \%$ body weight. Thus, the persisting effect of previous motivational level is not dependent upon method of weight maintenance. As suggested by others (e.g., Zaretsky, 1966), this persisting effect of previous motivational level could be taken to indicate that motivational level influences learning in some manner.

It was also found here that animals maintained by the FP method increased in speed over days, a result obtained previously by others in the activity wheel (Moskowitz, 1959), the Skinner box (Davenport \& Goulet, 1964, Marwine \& Collier, 1971), and the straight alley in some cases (Ehrenfreund, 1960, Figs. 2 and 3). In contrast to Marwine and Collier's results, this increase in performance was not related to a decreasing ration. This difference in results could be related to any number of differences in procedure between the two studies, e.g., the missing nutrient (food vs water), percentage body weight (95\% here vs $75 \%$ in Marwine and Collier's study), or performance measure (rate of response vs speed).

While animals maintained by the FP method increased in speed over days, animals maintained by the AP method maintained a constant speed. There are at least two different interpretations of this difference. If it is assumed that all groups were at asymptote at the end of Phase 1, then the increase in speed for the FP groups may be attributed to increasing motivation, while the constant speed of the AP groups may be assumed to reflect a constant motivational level. Alternatively, it could be assumed that the groups were not at asymptote by the end of Phase 1, and thus the FP groups increase in speed in Phase 2 because they are still learning, their motivation being constant, while the AP groups maintain a constant speed because they are still learning, and their motivation is decreasing - two factors which balance each other.
Other interpretations are also possible Thus, the present results are indeterminate regarding the question of which method of weight maintenance produces a more constant motivational level.

\section{REFERENCES}

DAVENPORT, D. G.. \& GOULET, L. R Motivational artifact in standard food-deprivation schedules. Journal of Comparative \& Physiological Psychology $1964,57,237-240$.

EHRENFREUND, D. The motivational effects of a continuous weight loss schedule. Psychological Reports, 1960, 6 , 339-345

MARWINE, A. G., \& COLLIER, G. Instrumental and consummatory behavior as a function of rate of weight loss and weight maintenance schedule. Journal of Comparative \& Physiological Psychology. $1971,74,441-447$

MOSKOWITZ, M. J. Running-wheel activity in the white rat as a function of combined food and water deprivation. Journal of Comparative \& Physiological Psychology, 1959, 52,621-625.

TRAUPMANN $K$ L Acquisition and extinction of an instrumental response with single- or multiple-pellet riward. Psychonomic Seitence, 1971, 22,61-63.

ZARETSKY H H Letning and performance in the runway as a function of the shift in drive and incentive. Journal of Comparative \& Physiological Psychology, 1966,62, 218-222. 